\title{
Results of Theoretical and Experimental Researches of Anomalous Low Friction and Wear in Tribosystems
}

\author{
Viacheslav Stadnychenko, ${ }^{1,}$, Valeriy Varvarov ${ }^{2}$ \\ ${ }^{1}$ Department of Hydro and Gas Systems, National Aviation University, Kyiv, Ukraine \\ ${ }^{2}$ Faculty of Aviation Engineering, Ivan Kozhedub Kharkiv National Air Forces University, Kharkov, Ukraine
}

Email address:

stadnychenko1971@gmail.com (V. Stadnychenko)

${ }^{*}$ Corresponding author

\section{To cite this article:}

Viacheslav Stadnychenko, Valeriy Varvarov. Results of Theoretical and Experimental Researches of Anomalous Low Friction and Wear in Tribosystems. Advances in Materials. Vol. 8, No. 4, 2019, pp. 156-165. doi: 10.11648/j.am.20190804.14

Received: August 30, 2019; Accepted: October 7, 2019; Published: October 25, 2019

\begin{abstract}
The paper presents results of theoretical and experimental researches of anomalously low friction from the standpoint of thermodynamics of non-equilibrium processes occurring under conditions of elastic interaction of microrelief of tribosystem elements. The search of theoretical prerequisites for the transfer of external friction from the "normal mode" to "abnormally low friction mode" is a timely and urgent task which this article addressed to. The conditions for achieving of the abnormally low friction and wear are the creation of the most favorable conditions for microcontact quasi-elastic interaction arising at the microrelief level. The rheological and structural conditions for the transformation of the external input energy by the contact interaction of the roughness of the surface layer of tribosystems are presented. Research have shown that the rheological properties of the surface layer are the main reason for the transition of tribosystems to abnormally low friction and wear mode. The analysis of the contact interaction of the roughnesses by the model of solid body in the form of interatomic bonds made possible to estimate the elastic energy which is released during the destruction of the bonds due to the coupling of the microroughnesses. The evaluation of the interaction as a Markov process with two bilateral states (decay and recovery) made possible to obtain a mathematical dependence of the generalized component of the impulse of force on the contact spot in the coupling state and the parameter of the distribution of contact spots by the modulus of canonical distribution of contact energy on the contact line.
\end{abstract}

Keywords: Anomalous Low Friction and Wear, Tribosystem, The Molecular-Mechanical and Wave Theories, Wave Component of Friction Force, Rheology, Kinetic Interaction of the Roughnesses, Acoustic Emission

\section{Introduction}

Recent advances in structural and energy and the molecular-mechanical theories of friction and wear allow us to consider tribosystem as a generator that converts mechanical energy into heat $[1,2]$. Thus, the results of calorimetric tests show that above 95\% energy input to tribosystem is converted into heat, and about $5 \%$ is accumulated in the surface layer and further provides the structural transformation, and partly is dissipated through fracture channel that is accompanied by acoustic, electromagnetic and other kinds of radiation [1, 3, 4]. All activities aimed at increasing durability of tribosystems are directly or indirectly pointed at improving the efficiency of the specified heat generator; and two main trends were identified in this area. The first-there is achieved reduction of the part of energy spent on destruction of the surface layer by improving the lubrication, structures of materials, application of various kinds of wear-resistant coatings [1]. The secondimproving of contact interaction conditions, reduction of friction coefficient and, consequently, decrease in absolute value of external energy input to the tribosystem $[5,6]$.

\section{Analysis of Main Achievements and Publications}

The strength of the external friction is directed against the movement in tribosystem and, in accordance with the 
molecular-mechanical theory of friction and abrasion, consists of two components: a molecular (adhesion) $F_{a}$ and mechanical (deformation) $F_{d}$ [2]. These components of the frictional force $F$ are being "in conflict" with each other when solid bodies are moving.

$$
F_{m p}=F_{a}+F_{d}
$$

When using finishing technique for burnishing of surface, it is possible to reduce significantly mechanical component of the frictional force. But in the same proportions the molecular component is increasing, and that triggers the adhesion-cohesive destruction of the surface layer of tribosystem at the breaking-in stage. As a result of breakingin the equilibrium state is formed, which complies with the friction conditions (force, sliding speed, temperature, etc.). This, in turn, defines the amount of external energy, which is supplied to the tribosystem and, as a result, determines the rate of formation and destruction of secondary structures on the friction surfaces, i.e. their wear resistance [1].

Compilation of a large number of interfacial friction study results shows that the value of the interfacial friction coefficients for different combinations of lubricant medium materials is in the range $0.005 \ldots .0 .02$, according to GercyStribeck curve [7].

\section{Research Purpose}

Thus, in the most common modes of friction and lubrication, both the surface layer of tribosystem elements and lubricant medium participate in formation of the frictional force. Currently, a number of studies present the results of experimental studies with indicators of the frictional force that are far beyond these bounds towards their reduction [8-11]. Therefore, the search for theoretical assumptions concerning the external friction transfer from a "normal area" to anomalous low friction is a timely and urgent task, the solution of which this article is focused on.

\section{Presentation of the Basic Material}

At analyzing this problem, it is very interesting to consider studies of the English scientist James Gray, who in the 30s found that the supply of dolphin's muscular energy constitutes only $10 \%$ of the estimated energy required to move with the observed speed $(10 \mathrm{~m} / \mathrm{s})$. That is, according to the laws of hydrodynamics the dolphin cannot move in the water with such a speed because of the small margin of its muscular energy, but, nevertheless, it does move-and this is the essence of the science famous "Gray's paradox."

Over the time there were proposed many hypotheses for explaining the reasons for the low resistance to dolphin's movement, which were based on the dolphin's skin elasticity. Experimental hydrodynamic tests gave negative results in this direction. Sir James Gray had provided himself invaluable assistance in discovering the physical mechanism of the anomalously low friction at the dolphin moving. $\mathrm{He}$ hypothetically suggested that between the nose of dolphin and the counter surface of the water there somehow emerged in motion and existed constantly the negative hydrodynamic pressure gradient, i.e. the dolphin on the move seeming to catch up oncoming water that is constantly running away from it.

To implement such a character of the objects interaction at their moving in a fluid, the authors of [12] developed the technology, the essence of which was that the reduction of hydrodynamic resistance during motion of a vessel was achieved by creating elastic compression waves in the liquid from the nasal surface in the opposite direction. Implementation of this approach in the modern shipbuilding makes it possible to increase by more than twice their rate of sailing at the modern capacity of engines and the same fuel consumption. Similar results on the frictional force reduction were achieved at development of tribological recovery technologies $[4,13]$. In this case, the effect of transition to anomalous low wear and friction was achieved due to mobility of bilayered metal-ceramic layer formed on the friction surfaces, and in these studies for the first time the wave approach was used for explaining dissipation process for the energy supplied from the outside.

Of course, taking into account a significant difference in the hydrodynamic and external friction when moving most real tribosystems operating under boundary friction conditions, the use of these technologies to achieve an anomalous low friction condition is practically impossible. However, the wave nature of this phenomenon (Gray's paradox) allows to put forth a scientific hypothesis that in addition of molecular and mechanical components of frictional forces, there is a wave component $F_{w}$ :

$$
F_{m p}=F_{a}+F_{d}-F_{w}
$$

Under ordinary conditions of boundary friction, due to interaction of the lubricating medium with plastically deformable materials of triboelements there occurs a "softening" of their surface layer (Rehbinder effect) [7]. Positive gradient of the surface layer hardness is formed in depth. Therefore, the tribosystem passes from elastic contact to elastic-plastic and plastic contacts. The frictional force thus reduced to a steady-state value. The magnitude of the frictional force for the given conditions of contact interaction in the tribosystem is determined by the first two components of expression (2). The wave component $F_{w}$ in this case is negligible, and therefore was not taken into account up to now, though the wave component of the friction was pointed out, both in the past and in the present. So, I. Kragelskiy, while explaining elastic deformation of the material by friction, has revealed that the contact points produced the elastic deformation of the material, while at coming out of engagement the contacts started to oscillate, i.e. practically the wave component of the frictional force was formed [2].

The results of studies of the elastic contact at friction revealed that under certain circumstances the oscillations could be an additional channel for dissipation of the energy 
supplied to the tribosystem $[14,15]$. Conditions for formation of real tribosystems that work exclusively in the elastic zone are set out in [7, 15-17]. Theoretical basis for the use of wave effects to achieve an anomalous low friction and wear is the work of A. Veynik, who on the basis of non-equilibrium thermodynamics has formulated the conditions for achieving both zero and negative external friction [14].

Through analysis of reversible and irreversible processes, the author of this study concluded that the magnitude of work of the frictional force could not be regarded as a criterion for assessing the irreversibility of the process ("irreversibility factor"). The concept of an irreversible process in friction is purely conditional, since under irreversibility there was understand, since the time of Clausius, the inexorable onesidedness course of all real processes. In fact, according to A. Veynik in nature the processes with minus-friction are as widespread as plus-friction processes.

If we consider the interaction of microroughnesses in the contact area of tribosystem, by analogy with the example from [15], then the effect of the conversion of mechanical energy into thermal energy $Q_{D}$ in the tribosystem at elastic interaction of microroughnesses under conditions of equality of interacting masses (microroughnesses on the surface) of the movable and immobile friction bodies can be estimated by the following expression:

$$
Q_{D}=(1 / 2) \delta P_{m D} m=(1 / 2) m\left(\omega_{c}^{2}-\omega^{2}\right)
$$

where $\delta P_{m D}$ - the difference between the kinetic energies of the interacting microroughnesses; $m$-connectable (disconnectable) mass of the microroughnesses; $\omega_{c}$-initial speed; $\omega$-final speed.

At elastic impact of the microroughnesses $Q_{D}$ depends on degree of the impact perfection, which is estimated by losses on plastic deformation or destruction. Under conditions of absolute elastic collision of bodies with fixed obstacle the mass velocity before impact is equal to its velocity after impact. Brakeage of the mass is accompanied by dissipation heat release in amount $Q_{D}$, while its acceleration to the previous velocity-by absorption of the same amount of heat$Q_{D}$. As a result, the shielding effect (dissipation) becomes zero. This is followed from (3)-at entering in it the total flow of the kinetic component of the total energy of the body it will be zero. However, the presence in (3) of difference of squared velocities demonstrates the critical importance of the fact, which of the velocities is more-initial or final. If the initial velocity $\omega_{c}$ at impact is more than the final velocity $\omega$, i.e. the activity of kinetic component of the total energy of the body decreases as a result of the shielding effect, then the heat of dissipation is positive, it is released (plus friction). If the initial velocity $\omega_{c}$ is less than final velocity $\omega$, i.e. the activity of kinetic component of the total energy of the body increases as a result of the shielding effect, then the heat of dissipation is negative, it is absorbed (minus friction).

In view of the above, the surface energetic does not allow considering the roughness only from the geometric point of view. In the theory of dissipation, it is known an interesting physical effect of Benard cells formation [18]. If the surface is constantly supplied with energy, then, when it reaches a certain value, there must be provided more intense its recoil (scattering) into environment. There arises bifurcation followed by self-organization of the surface structure, and at that, the surface is divided spontaneously into separate elements, each of which dissipates more extensively than before the bifurcation [18]. Let us analyze the energy exchange between the rubbing surfaces by approximating irregularities in the form of energy cells, each of which gives energy to the counterbody, and takes it from the counterbody. This energy exchange is estimated at the energy flows averaged over the whole surface. If we look locally at these flows, then depending on the elastic-plastic properties of triboelements there also become achievable conditions of plus and minus friction with consideration for interrelation between molecular-mechanical and wave components of the frictional force.

Thus, if the actual tribosystem is represented as a number of interacting elastic microroughnesses, the main reason for minimizing the magnitude of the tribological parameters (rate of wear and temperature in the contact area, frictional force, etc.) will be a kinetic nanofield (reaction of microroughnesses' masses to external impact that appears in local points of elastic interaction of the rubbing bodies). At that it is possible to achieve the conditions when both the effects-dissipation and anti-dissipation-are strictly equal to each other because the work of nanofield forces is always equal to the work of deceleration or acceleration of the masses, but the signs of these works are antithetical.

Amount of dissipative component $d \Theta_{D}$-the heat evolved or absorbed is directly proportional to a change in activity of the kinetic component of nanofield $d \Theta_{D}$, and to the magnitude of heat transferred as a result of local interactions $d E$, and inversely proportional to absolute temperature of the medium $T$, which can be described by stochastic equations, the resulting solutions of which can be represented by [20]:

$$
d \Theta_{D}=d Q_{D} / T=-d P_{D} d E / T
$$

Currently, when assessing the state of stress of the surface layer, they manipulate with levels of the stresses of certain signs, compressive stresses or tensile stresses at the whole effective scope of interaction, which are balanced in the cross-section of contacting bodies.

In the case of kinetic nanofield, the value of local stresses can be represented by cavitation effect in fluid, when at the same time there exist micro areas with high and low pressure, the total stress is a time-dependent value. If these conditions are realized for the tribosystem, the total change of temperature, frictional force and wear during the load change in the contact area will also be close to zero.

Thus, hypothesis of quasi-elastic interaction of surfaces followed by effects of the zero and minus friction contains the prospects for development, both in theory and in solution 
of the main application issue on minimizing friction and wear for a wide range of vehicles and machinery tribosystems [6, 14].

The existence of friction and anti-friction effects, dissipation and anti-dissipation makes a fresh look at the problem of irreversibility in tribosystems, and on this basis to develop a fundamental approach to reducing friction and wear of real tribosystems [20]. Self-regulation for this kind of tribosystems is carried out via the channel of excessive entropy production, which determines the tribosystem stability under non-equilibrium self-organization [17]. Changing of the growth of excessive entropy production $\left(\delta^{2} S\right)_{m c}$ in time for non-equilibrium processes described above is of oscillatory character. So, the sign will determine the interrelation of the dissipation and anti-dissipation processes.

For analysis of the tribosystem self-organization processes there are very important not only the kinetic interactions arising in the submicroscopic and more subtle level, but also the behavior of the surface layer after the tribosystem stoppage. The tribosystem stoppage leads to the exclusion of the boundary layer disarrangement, while processes directed at the self-organization still continue to operate during some time, resulting in further collating of the frictional contact and, as a consequence,-reducing the frictional force under repeated loading [21]. This allows to change significantly the methodology of automatically operated loading aimed at transferring the tribosystem to a higher level of selforganization at its derivation beyond the stability limits. The automatically-operated loading provides complete correspondence between the growth of the applied load and inner stresses relaxation rate due to behavior of diffusive and microshift processes. After automatically-operated loading the metal becomes more uniform as to character of stresses distribution. The structure of automatically-strengthened materials is mechanically more equilibrium as compared to initial material, and has improved resistance to commence of plastic deformation and simultaneously-reduced liability to brittle fracture [16].

At the same time, the impulsive loading applied to the tribosystem of the preset structured state causes the spontaneous transition of different levels of movement of plastic deformation of substructural microlevel to the mesolevel that substantially exceeds the depth of coldhardened layer, and at that there has been observed the abrupt change in properties of tribosystem materials. In this case, the behavior of materials at external mechanical impact is usually regarded as anywhere medium between crystal and liquid states. Quasi-elastic layer is formed on the surface; hydrodynamic deformation is hypothetically possible in its central part, while on the periphery in the transition zone we should expect intensive rotary elastoplastic deformation, similar to the structure of vortex formation in the boundary layer of fluid flow [22]. Based on the studies of I. Prigozhin, A. Veynik in non-equilibrium self-organization area, we have conducted two series of experimental studies. The general idea of the studies was to conduct a breaking-in at the first stage of tribosystem activity, to achieve the equilibrium self- ogranization conditions in tribosystem, which are characterized by constant values of: surface average temperature, force of friction and wear rate. At the second stage the tribosystem was transferred above the stability limit due to mechanical energy input. These conditions were created due to impulsive (automatically-operated) loading (Figure 1) [6].

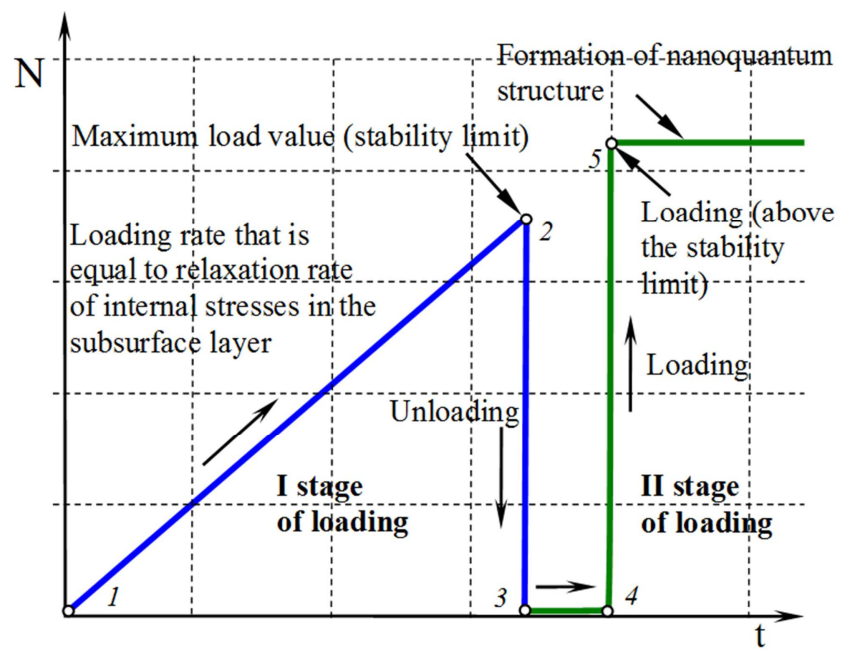

Figure 1. Automatically-operated loading at the tribosystem transfer to a higher level of non-equilibrium self-ogranization: 1-beginning of the loading; 2 -stability limit at transition to damage; 3, 4-achieving the maximum uniform distribution of stresses in the surface layer; 4-impulsive loading; 5-bifurcation point of tribosystem transfer to a higher level of selfogranization.

As a result of automatically-operated impulsive loading the gradient law of hardness variation in the surface layer changes from positive (after Kragelskiy) to negative one (Figure 2).

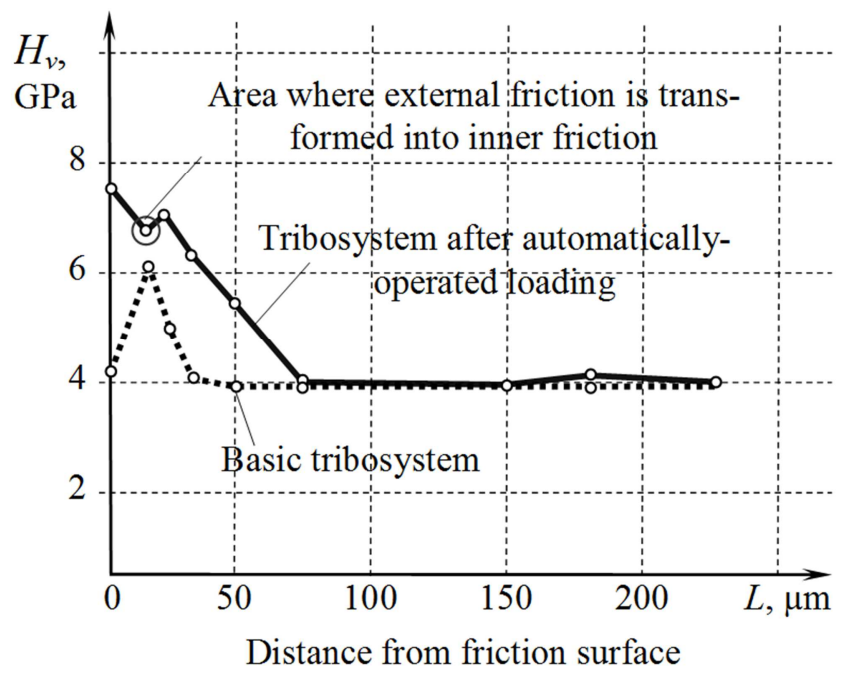

Figure 2. Pattern of microhardness $H_{v}$ change in depth of the surface layer of the fixed element of tribosystem "Steel 30HGSNA-Steel 30HGSNA" (30XГCHA) after the breaking-in (loading mode: $P=1000 \mathrm{H}, \mathrm{V}=500 \mathrm{~min}^{-1}$; the operating environment $M 10 G_{2 K}$ ) and impulsive automatically-operated loading at conducting friction and wear tests.

Significant reinforcement both in magnitude and in the 
depth of the surface layer can be explained on the basis of this study. Friction, as a process of rapid transformation of mechanical energy into internal energy, is one of the most powerful sources of impulsive impact on materials due to its specific characteristics. It is found that with increasing of deformation degree there takes place a significant increase of dislocations density, resulting in significant decrease of residual grain size. When the magnitude of the relative strain $\varepsilon \geq 10$, the grinding of grain structure occurs up to the nanoscale (below $100 \mathrm{~nm}$ ) [23, 24].

The principles of nanostructuring in the conditions of transition to abnormally low friction and wear have been developed in the works of S. V. Fedorov [8-10]. For the analysis of anomalously low friction, a quantum mechanical approach was used, the concept of a mechanical quantum was introduced - the minimum number of atoms capable of providing a configurational distribution of nanostructures having the properties of the back-sensing and dissipating (rotating) energy of external mechanical motion. It is also the smallest structural formation under conditions of plastic deformation, and it is formed during the transition of tribosystem (deformed volume) through the boundary activated (critical) state due to the development of selforganizing processes of tribosystem adaptation. In the volume of tribosystem, under conditions of abnormally low friction and wear (elemental tribosystem), the number of such mechanical quanta (tribosystems) $\widehat{\varepsilon}$, a safe number of fatigue cycles, is $0,63 \cdot 10^{8}$. The mechanical quantum itself is a dynamic oscillator of dissipative friction structures and its linear size is equal to the radius of the spherical ideal crystal 7,177 Nm [8-10].

In view of the above stated, automatically-operated loading creates new conditions for the surface roughness formation. If at normal breaking-in the roughness is mainly formed as a result of wear of tribosystem elements, then at the automatically-operated loading the roughness is formed due to processes of the surface layer structuring, which is clearly seen in the micrographs of the friction surfaces.

Let us consider in more detail the changes of the tribological parameters through the example of tribosystem "steel-bronze". To conduct the automatically-operated loading, the friction machine $2070 \mathrm{CMT}-1$ was upgraded to regulate in a wide range the loading rate and to ensure possibility of tribosystem impulsive loading [26]. Research samples were made of steel, 30H3VA (30X3BA) and bronze VB23NTS (ВБ23HЦ). As a working fluid there was used aviation kerosene TS-1 (TC-1) with a flow rate of 3.5 liter per hour. Test conditions: load $600 \mathrm{~N}$ at operation under stationary conditions; drive shaft rotational speed-500 $\mathrm{min}^{-1}$; ambient temperature $+20^{\circ} \mathrm{C}$.

Automatically-operated loading was conducted in accordance with recommendations of the study [6]. The test samples were manufactured with high quality of the surface treatment. The wear rate was measured in real-time with the use of acoustic emission technique [20]. To compare the wear resistance of the investigated tribosystems there was used the average wear rate (ratio of the integrated value of the average power of acoustic emission to the number of measurements). The test results for the basic tribosystem and tribosystems subjected to the automatically-operated loading are depicted in Figures 3-5.

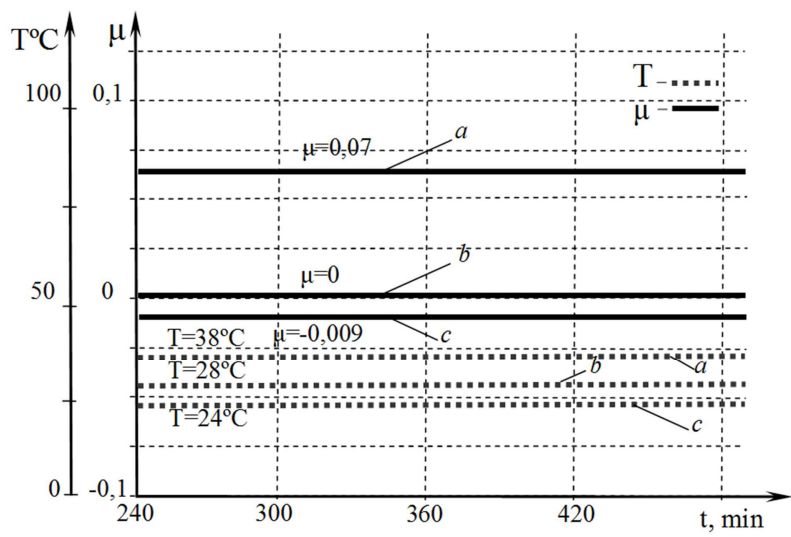

Figure 3. Tribotechnical characteristics of the studied tribosystems at the final stage of the operation under load of $600 \mathrm{~N}$ : a-basic tribosystem; $b$ tribosystem at the automatically-operated loading (impulsive load of 1400 $N$ ); c-tribosystem at the automatically-operated loading (impulsive load of $1800 \mathrm{~N})$.

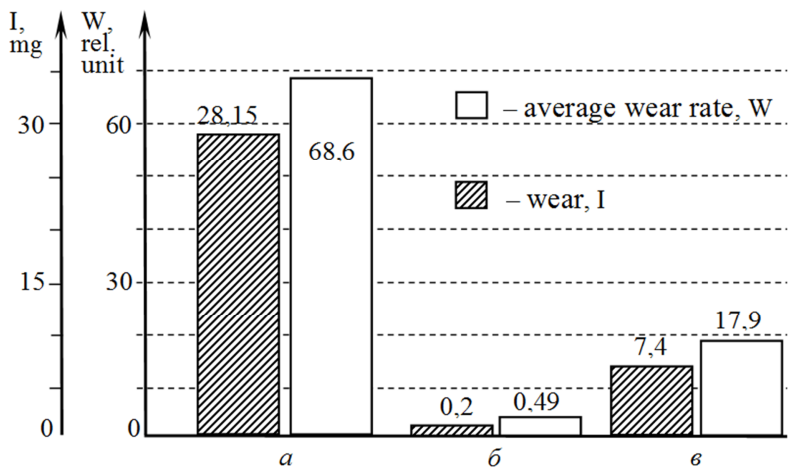

1-I, mg; 2-W, rel. unit; 3 -average wear rate, W; 4-wear, 1

Figure 4. Total wear of tribosystem 30H3VA (30X3BA)-VB23NTS (BБ23HЦ) at 8-hour test: a-basic tribosystem; b-tribosystem at the automatically-operated loading (impulsive load of $1400 \mathrm{~N}$ ); c-tribosystem at the automatically-operated loading (impulsive load of $1800 \mathrm{~N}$ ).

The test program included conducting of basic wearability tests with the definition of basic tribological parameters under the load of $600 \mathrm{~N}$. The second test series included breaking-in at this load and the tribosystem transfer from equilibrium to non-equilibrium friction as a result of automatically-operated loading. The value of impulsive loading under the program amounted $1400 \mathrm{~N}$ and $1800 \mathrm{~N}$, with a transition on the final loading stage to the operational load of $600 \mathrm{~N}$.

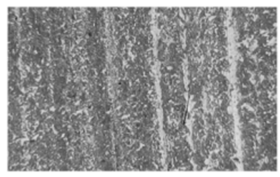

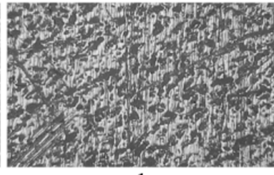

$\mathrm{b}$

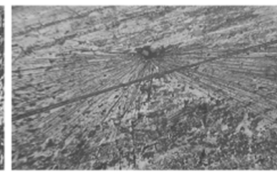

$\mathrm{c}$
Figure 5. Surface fractography of bronze triboelements VB23NTS $\times 250:$ abasic tribosystem; b-tribosystem at the automatically-operated loading (impulsive load of $1400 \mathrm{~N}$ ); c-tribosystem at the automatically-operated 
loading (impulsive load of $1800 \mathrm{~N}$ ).

Analysis of the results revealed that transition of the tribosystem operation in the mode of anomalous low friction and wear had quite good repeatability. This article presents results of experimental study of friction pairs 30H3VAVB23NTS (30X3ВA-ВБ23НЦ) in the working fluid of aviation kerosene TS-1 (TC-1).

Let us analyze the results of the anomalous low friction resulting due to automatically-operated impulsive loading up to $1400 \mathrm{~N}$, Figures 2-4 b, based on local kinetic contact interaction in tribosystem. That is, let us consider the physics of kinetic nanofield formation at the elementary elastic contact of microroughnesses under the external friction.

The most natural explanation of the microroughnesses effective volume interaction is it representation as a Markov process with two discrete states, in particular the "Bilateral reaction", which offers the opportunity of only $1 \leftrightarrow 2$ transition in the system (1-state $x_{k}$-recovery, 2-state $x_{j}$ dissociation), (e.g. deformation and recovery of the original shape of the effective volume).

A. Kolmogorov equation is hold for this process [27]:

$$
\frac{\partial p_{1}\left(t_{1} x_{k}\right)}{\partial t}=\sum_{i} A_{j k}(t) p_{1}\left(t_{1} x_{j}\right)
$$

where $p_{1}\left(t_{1} x_{k}\right)$-one-dimensional probability of operative condition;

$$
A_{j k}=\left[\frac{\partial p\left(u_{1} x_{k} / t_{1} x_{j}\right)}{\partial u}\right]_{u=t}
$$

$p\left(u_{1} x_{k} / t_{1} x_{j}\right)$-probability of transition from state $x_{k}$ to state $x_{j}$, so, given the normalizing

$$
A_{j k}(t) \geq 0(j \neq k) ; A_{i j}(t)=-\sum_{k \neq 1} A_{j k} \leq 0 ; \sum_{k} A_{j k}(t)=0
$$

With regard to a great number of local interactions of microroughnesses considered under both theoretical and experimental approach, the probability of finding an elementary contact in one of the states of the two-stage model depends on the state at the last time point $t_{n}$ and does not depend on history. Solution of previously formulated equation is a Markov diffusion process. It is obvious that to solve it we need to impose restrictions on the right side of the equation and to assume, in particular, that the load vector $q(t)$ is delta-correlated in time process. This condition is achieved by carrying out experimental research with the use of automatically-operated loading.

When impulsive loading is supplied at the final stage of the automatically-operated loading, a powerful specific concentration of energy on microcontacts arises, resulting in "magma-plasma" condition of the material with inhered special properties $[8,24]$.
In this case, conditions of non-equilibrium selfogranization are accompanied by processes, inconsistent with the traditional principles of thermodynamics of equilibrium systems; in particular, in the case there occurs a state of the entropy auto regulation due to processes of fluctuations symmetry in accordance with the principles of least action [25].

On entering the designations $p_{1}(t, 1)=p_{1}(t)$-the probability of existence of the 1 -state of the system 1 (recovery), assuming the process is uniform over time, and $\alpha, \beta$-constant values: $A_{12}=\alpha$; $A_{21}=\beta ; \alpha+\beta=\lambda$, where $\alpha \partial t ; \beta \partial t$-probabilities of transition $1 \leftrightarrow 2$ (dissociation) and $2 \leftrightarrow 1$ (recovery) for the time $\partial t$, the equation (5) can be written as a system

$$
\frac{\partial P_{1}}{\partial t}=-\alpha p_{1}+\beta p_{2} ; \frac{\partial P_{2}}{\partial t}=-\alpha p_{1}-\beta p_{2}
$$

By solving equation (5) with the initial state $t=0, p_{1}=1$ (recovery), we obtain

$$
\begin{array}{r}
p_{1}(t)=e^{-\lambda t}+\beta / \lambda\left(1-e^{-\lambda t}\right) \\
p_{2}(t)=\beta / \lambda\left(1-e^{-\lambda t}\right)
\end{array}
$$

The two-stage model, while identifying the 1-state (recovery) with a contact in the coupling condition, and the 2-state (breakdown of link) as the sleeping contact (7) and (8), can be interpreted as a probability of such contacts being in these states or as a relative number of contacts that are in the system at any given time point $t$.

Thus, evaluating the stationary and therefore ergodicity, considering microcontact models of roughness model according to expressions (7) and (8), it is apparent that when $t \rightarrow \infty$ this equation has stationary solutions:

$$
p_{1}(\infty)=\beta / \lambda ; p_{2}(\infty)=\beta / \lambda
$$

Insomuch as the probability $p_{1}(t)$ is proportional to the number of contacts that are in the coupling condition (if we introduce the elementary force of coupling friction accrued to one contact), then expression (7) is similar to functional dependence of the static frictional force $F_{\text {frst }}$ (and of friction coefficient $\mu$ ) on the duration of the fixed contact set out by I. Kragelskiy:

$$
F_{f r s t}=F_{\infty}-\left(F_{\infty}-F_{0}\right) e^{-v t}=F_{0} e^{-v t}+F_{\infty}\left(1-e^{-v t}\right)
$$

where $F_{\infty}$-the frictional force corresponding to the infinite duration of contact, $F_{0}$-the frictional force at zero time of the contact.

In close connection with 2-stage model, we offer probabilistic interpretation of velocity function of force initiating pry out of the slipper from the surface of a rotating disk as a condition of bilayered metal-ceramic layer 
formation with its mobility under certain conditions of contact interaction [5].

Thus, to assess the relative number (probability) $P$ of the links that exist in the system (and are breakable when they slip from the surface of perfectly elastic body at a constant sliding velocity $v$ ), there can be written:

$$
P=P_{01} e^{\alpha v}+P_{02}\left(1-e^{-\beta v}\right)
$$

where, $P_{01}, P_{02}$-relative number of microroughnesses being in each state at $v=0, v=\infty$ respectively; $\alpha, \beta$. coefficients.

Comparing (10) with (7) after transition to friction coefficient $\mu$, we get:

$$
\mu_{\infty}=\frac{\beta}{\lambda}=\frac{\beta}{\alpha+\beta}
$$

Equation (8) relates to the frictional force at movement. At the number of states of the model with a large number of microcontacts, the equation contains oscillatory solution, which certainly must be accompanied by decrease of the frictional force.

Considering the difference in height of the microroughnesses, when moving there is formed a discrete sequence of the frictional force impulses with random amplitudes $\alpha v$ and moments of occurrence $t v$ corresponding to elementary displacements of interacting microscopic zones of rubbing surfaces. On the basis of this system of impulses that can be approximated, for example, with $\delta$-pulses you can construct an analog random function $x(t)$ and corresponding random process by averaging over a sufficiently large time intervals $\partial t^{\prime}$ of duration that exceeds the time between individual elementary pulses:

$$
x(t)=\int \sum_{v} \alpha_{v} \delta\left(t^{\prime}-t\right) \partial t^{\prime}
$$

Physically this corresponds to fixing the resulting displacements (elastic wave) at sufficiently large microstrip lines of rubbing surfaces. In turn, this random process averaged over time intervals can be approximated by Markov process, i.e. we assume that its distribution function $v\left(t_{1} x / t_{0} x_{0}\right)$ is described by Fokker-Planck equation:

$$
\frac{\partial v}{\partial t}=\frac{\partial A\left(x_{1} t\right) v}{\partial x}+\frac{1}{2} \frac{\partial^{2} B\left(x_{1} t\right) v}{\partial x^{2}}
$$

where $A, B$-coefficients.

Next, we introduce the conditional mean value $\bar{x}(t)$ of the displacements, but for the process (13), under the assumption that at the time point $t_{0}$ :

$$
\bar{x}=x_{0} ; \bar{x}(t)=\int_{-\infty}^{+\infty} x v\left(t_{1} x / t_{0} x_{0}\right) d x
$$

Now we construct a phenomenological equation of motion:

$$
\int_{-\infty}^{+\infty} A v d x=A\left(\overline{x_{1} t}\right)
$$

by multiplying the equation (13) by $x$ and integrating over $x$ within limits from $-\infty$ to $\infty$, suggesting that the integrated terms go to zero in the infinity.

At that, the equation (16) coincides with the common dynamic equation for a certain material point under condition:

$$
A\left(\overline{x_{1} t}\right)=a(t) \bar{x}+b(t)
$$

Thus, the Fokker-Planck-Kolmogorov equation is equivalent to the stochastic dynamic equation, i.e. use in physical and statistical interpretation of the stochastic equation to describe the motion of effective interaction volume that is dwell in the coupling condition, as well as the partition of the acting on it stochastic reaction force on the averaged and the fluctuating parts within this framework, seemed to be understandable and acceptable. At the same time the effective mass can be interpreted simply as a proportionality factor included in $A\left(\overline{x_{1} t}\right)$. Given the connection between equations (13) and (16), we can formulate the meaning of exponential distribution on energies for the system of microcontacts that are in the coupling condition.

Since there exists a stationary solution of equation (14) $\partial v / \partial t=0$, then:

$$
j=-A v+\frac{1}{2} \frac{\partial B v}{\partial x}=\text { const }
$$

Stationary flow $j$ on the boundary $x$ of the domain of variation is assumed to be zero, then after integration:

$$
v(x)=\frac{C}{B(x)} e^{\int^{x} \frac{A(S)}{2 B(S)} d s}
$$

where $C$-const, determined from the condition of standardization. If at constants $A, B$ we assume:

$$
\int_{0}^{x(t)} \frac{A(S)}{2 B(S)} d S=\frac{A x}{2 B}=-\frac{\Delta E_{w}}{\Theta}
$$

where $\Delta E_{w}$ - the energy of contact under coupling condition; $\Theta$-module of canonical distribution.

Consider the energy conversion $\Delta E_{w}$ in contact interaction more detailed. In the process of coupling, an elastic wave is generated at each elemental contact. According to quantum mechanics, the energy and impulse associated with each normal oscillation (with each wave) are edged, it means that they can take only discrete values. They become multiples ( $\mathrm{n}$ 
$=0,1,2 \ldots$ ) by the equations of Louis de Broglie (dualism of energy and impulse of a moving particle) [26], which take on the physical meaning, respectively, of the energy and impulse of the "elemental excitation" of oscillatory motion in a crystal. Such elemental excitation can be regarded as a quasiparticle (energy defect of a rigid body) with a quasiimpulse $\vec{v}$ and energy

$$
\varepsilon(\vec{v})=\hbar \omega(\vec{v} / \hbar)
$$

where $\varepsilon$-the energy of the moving particle;

$\vec{v}$-impulse of motion of a particle;

$\hbar$-Plank constant;

$\omega$-wave oscillation frequency.

These quasiparticles are called phonons. They are the simplest type of elementary excitations in solids [27]. Taking into account the accepted structure of the surface under conditions of abnormally low friction and wear, the dissipation energy of each mechanical quantum is equal to:

$$
\Delta E_{M Q}=n(\hbar \omega(\vec{v} / \hbar))
$$

where $n$-the degree of excitation of normal oscillation. This is the number of phonons with impulse $\hbar \vec{q}$; where $\vec{q}$-wave vector which direction coincides with wave and energy propagation direction $\hbar \omega$.

For the whole tribosystem which operates under the conditions abnormally low friction and wear, its dissipation energy is equal to:

$$
\Delta E_{T S}=\Delta E_{M Q} \widehat{\varepsilon}
$$

where $\widehat{\varepsilon}$-the number of mechanical quanta involved in the interaction.

As $\mathrm{n}$ - any integer, phonons, like photons, obey BoseEinstein statistics.

Using this approach, the function of the distribution of impulses of friction force on local spots is approximated by a Markov process and is described by the Fokker-Planck equation. On the basis of the solution of this equation the expression of the resultant impulse of the friction force for stationary conditions of tribosystem operation under conditions of abnormally low friction and wear is obtained. The expression of the impulse of force in this case does not reflect self-regulation when achieving equality between the molecular-mechanical and wave components of the friction forces at changing external conditions, for example, when the load changes.

This why equation for impulse $\vec{v}_{T S}$ of friction force of tribosystem is:

$$
\vec{v}_{T S}=e^{-\frac{\Delta E_{M Q} \hat{\varepsilon}}{\Theta}}
$$

Distribution (24) is a kind of equivalent to (19), however not for microdisplacements system described by equation (15), but for the system of traveling wave induced by them in the surface layers, which converts an external friction caused by molecular and mechanical components at the point of contact into the inner friction.

Value of the constant is determined by the physical and mechanical properties and by the structure of tribosystem elements causing migration by deformation waves of the most of the energy generated at local dynamic interaction. It is logical to assume the existence of certain critical speed $v(x)$, at which this balance is disturbed that causes to instant destruction of material. This hypothesis is supported by studies of energy transfer mechanisms under shock loading of materials due to propagation of elastic and plastic deformation waves [28]. In the study of the propagation of shock waves in continuous media, the authors used hydrodynamic theory, according to which the shock front is considered as a surface of discontinuity of the functions that describe the particles motion before and behind the shock front. For description of the materials, behavior under these conditions there were used equations of change in momentum, of mass balance and the flow of energy at motion of the functions discontinuity surface in a heterogeneous solid medium. The functional dependence of the critical speed $v_{c r}$ on the energy expended for deformation of a unit volume of material $E_{\text {un }}$ in this case is approximated by the expression:

$$
v_{c r}=\left(2 \mathrm{kE}_{\mathrm{un}} / \rho\right)^{\frac{1}{2}}
$$

where $\mathrm{k}$-coefficient of proportionality; $\rho$ - tension with account of "viscous" losses (internal friction).

Expression (25) is close in its physical meaning to the expression (24).

Above calculations are related to the longitudinal waves, as elastic oscillations in addition to longitudinal waves have a transverse component. Each component of the oscillations that extend into the interior of triboelements is a standing wave given the fact that at elastic oscillations of the values of local stresses must be of finite value in the whole volume [29]. Distributions of displacements form the volume sections that oscillate in opposite phases thus providing nullification of operating stresses and, as a consequence, form the amount of wear in the tribosystem.

In our opinion, the condition of anomalous low friction and wear is the equality of the energy components of the molecular-mechanical and wave components of the frictional forces. Violation of this balance (Figure 5, c) results in accumulation of defects in the subsurface area and formation of wear particles. The mechanism of the wear itself requires more detailed study with the use of modern metallophysical and physical methods. Further investigation of anomalous low friction of the tribosystem analyzed at different values of the impulsive loading magnitude increase at the final stage of the automatically-operated loading, which are two levels above $1800 \mathrm{~N}$ (Figures 3-5, c), has confirmed this hypothesis.

In this case, the tribosystem transfers to domain of negative friction, where the coefficient of friction $\mu=-0.035$ with the subsequent stabilization at a level $\mu=-0.009$ (Figure 3), which is associated with an 
imbalance between the absolute values of molecularmechanical and wave components of the friction. The physical aspects of this transition can be explained only from the position of non-equilibrium thermodynamics, after in-depth study of microstructural changes in the tribosystem elements on depth of the surface layer, and with the assistance of modern physical theories, which allow for a fresh look at the effect of the energy distribution symmetry on the mechanism of its transfer (superstring theory) [30].

When there is a negative friction, significant wear rate is observed, which is recorded by parameters of acoustic emission. The physics of this wear is correlated with internal processes of energy exchange in the subsurface area of the triboelement. The emergence of large wear particles on the friction surface occurs in the form of a microburst, Figure $5 \mathrm{c}$.

Thus, in accordance with the conclusions of A. Veynik, at nullification of the frictional force (see in Figures 3-5, b) there is no violation of the energy conservation law [14]. At the same time, violation of the energy balance between the molecular-mechanical and the wave components of friction forces, which arises at the negative friction, results in completely different physical mechanisms of the wear. According to fractographic studies, the wear particles are formed in the subsurface layer, they crack the intact surface layer, and at that when the wear particle leaves the area of contact it does not cause to formation of normal friction relief directed along the line of movement. The possible destruction mechanism of the wear in this case may be an unbalanced inner friction. However, this statement requires more detailed research.

\section{Conclusions}

Thus, the wave component is added to the molecular and mechanical components of the friction force at anomalous low wear, and, in this case, it is prevalent. As such, under conditions of anomalous low friction and wear, Gray paradox phenomenon is realized at a higher level of self-ogranization. The tribosystem transition from normal to anomalous low friction at the use of automatically-operated loading, in our view, generates a special kind of wave resonance, which arises in the kinetic nanofield with the formation of a traveling wave. Under the conditions of equilibrium selfogranization this effect does not occur, it is prevented by the principles of thermodynamics for equilibrium systems, especially due to formation of positive hardness gradient (Rehbinder effect).

Summarizing the studies performed, we can make a final conclusion that A. Veynik hypothesis about existence of the anomalous low, and even negative, friction is viable, it is confirmed experimentally, and has great practical significance. So, transition of tribosystem to zero friction under conditions of automatically-operated loading for "steel - bronze" tribosystem improves its wear resistance up to 140 times, in comparison with the base tribosystem, Figures 2-4, a. The wear, in this case, occurs at the initial stage of automatically-operated loading (during the breaking-in) and ceases at the final stage of the impulsive loading, which is confirmed by the results of monitoring the wear rate with the use of acoustic emission method.

\section{References}

[1] Ibatullin I. D. Kinetics of fatigue strength and distruction of serface layes: монография/I. D. Ibatullin.-Samara: Samara. state technical univercity, 2008.-387 p.

[2] Gromakovsky, D. G., Kovshov, A. G., Ibatullin, I. D., Dynnikov, A. V. Problems of Kinetics of Surface Destruction. Proceedings of VII-th International Symposium "INTERTRIBO 2002", section B-Wear, Slovak Republic, Stara Lesna, House of Technology, 2002, p. 57-58.

[3] Stadnychenko V. Synergetic Concept of Self-Ogranization in Tribological Systems at Heat Flow Managing/V. Stadnychenko, O. Troshin// Bulletin of Technical University "KhPI". Proceedings. Special issue: Technologies in Mechanic Engineering.-Kharkiv: NTU "KhPI»-2007.-No. 17-pp. 49-62.

[4] Tribology. Physical Basis, Mechanics and Engineering Applications/P. Berkovich, D. Gromakovskiy; edited by D. Gromakovskiy-Samara: Samara State Technical University, 2000.-p. 268.

[5] Zaporozhets' V. Mechanism of Energy Dissipation at Friction of Metal-Ceramic Layer in Technologies of Tribotehnichnical Recovery of Machine Parts and Mechanisms/V. Zaporozhets', V. Stadnychenko, O. Troshin// Weapon Inventory and Military Hardware.-Kharkiv: KhNUAF, 2010.-No. 2 (22).-pp. 113-118.

[6] Troshin O. Methodical Aspects of Non-equilibrium SelfOgranization of Tribosystems/O. Troshin, V. Stadnychenko, N. Stadnichenko et al.//Weapon Inventory and Military Hardware.Kharkiv: KhNUAF, 2014.-No. 1 (37).-pp. 233-241.

[7] Berkovich P. I. Tribology. Physical basics, mechanics and technical applications/P. I. Berkovich, D. G. Gromakovskiy; under reduction D. G. Gromakovskiy.-Samara: Samara state technical. university, 2000.-268 c.

[8] Fedorov S. V. Common patterns of friction evolution from the standpoint of self-organization and synergetic/S. V. Fedorov//Bulletin of science and education of North-West Russia (Scientific peer-reviewed electronic journal).Kaliningrad, 2016.-T. 2.-№ 1.-P. 1-14. [Electronic resource].Access: http://vestnik-nauki.ru.

[9] Fedorov S. V. Generalized Regularities of functional for tribological contacts. Antristechnisches Kolloqium 2017, ATK 2017: Marz 7-8, 2017-Aachen, Germany. Tagungsband. Institute fur Machinengestaltung, RWTH Aachen University, pp. 507-521.

[10] Fedorov S. V. Nano-Structural Standard of Friction and Wear//Tribology in Industry. 2018 Vol. 40 (2), pp. 225-238.

[11] Stadnichenko V. Resource Prediction for Tribosystems Operating in Nanowear Mode by Acoustic Emission Method/V. Stadnichenko, O. Troshin, A. Priymak et al.//Proceedings of KhNUAF.-Kharkiv: KhNUAF, 2010.-No. 4 (26).-pp. 41-48. 
[12] Pat. 2397101 C1 Russian Federation, IPC V63V 1/36 (2006.01) Device for Increasing the Velocity of Ship Sailing/A. Dzyuba; patenter: Dzyuba A.-No. 2009121794/11; Appl. 06/08/2009; Publ. 08/20/2010, Bull. No. 23.

[13] Pat. 41896 Ukraine, IPC V23R 6/00, S23S 24/00 S10M 125/30 (2009.01) Method of In-Place Recovery of Tribosystems Friction Surfaces/Voytov V., Stadnychenko V., Stadnychenko M., Bilyk A.; applicant and patenter Kharkiv Petro Vasylenko National Technical University of Agricultureu200901117; Appl. 02/12/2009; Publ. 06/10/2009, Bull. No. 11.

[14] Veynik A. Thermodynamic Couple/A. Veynik-Minsk: Nauka i Tehnika, 1973.-p. 284.

[15] Stadnichenko V. Metodology of Contact Interaction Processes Management Based on Acoustic Emission Effects in Tribotechnology Systems: Thesis Abstract... Dr. Techn. Sciences: 02.05.04/V. Stadnichenko; National Aviation University.-Kyiv, 2014.-p. 40.

[16] Gindin I. Physics of Automatically-Operated Hardening/I. Gindin, I. Neklyudov.-Kiev: Naukova Dumka, 1979.-p. 184.

[17] Vasiliev A. Directed Formation of Products Properties in Engineering/A. Vasiliev et al.-M.: Mashinostroenie, 2005.-p. 384.

[18] Prigojin I. Time, chaos, quantum. Toward a solution to the paradox of time/I. Prigojin, I. Stengers;-[Iss. 8-th].-M.: EDITORIAL 2014.-229 p.

[19] Zaporozhets V. Theoretical and Experimental Bases of Acoustic Emission Identification of Wear Mechanisms and Forecasting of Tribosystem Resource/V. Zaporozhets, V. Stadnichenko, O. Troshin//Problems of Tribology.Khmel'nyts'kyy: KhNU, 2013.-No. 1 (67).-pp. 89-98.

[20] Stadnichenko V. Classification of Nanowear Types by Value of Dissipation Factor of External Energy Supplied to Tribosystem/O. Troshin, V. Stadnichenko, N. Stadnichenko et al.// Proceedings of KhNUAF.-Kharkiv: KhNUAF, 2010.-No. 1 (27).-pp. 51-61.

[21] Baranov A. Self-Ogranization of Tribosystems at Boundary Friction of Metals/A. Baranov, V. Vagner, S. Tarasevich et
al.//Polzunovskiy vestnik. 2009.-No. 1-2.-pp. 155-158.

[22] Pogodaev L. Structural and Energetic Models of Behavior (Reliability) of Materials at Impulsive Loading/L. Pogodaev et al.// Friction, Wear, Lubrication.-2013. Vol. 15, No. 57. [Electron. resource].-Access: http://www.tribo.ru.

[23] Yurkova A., Belots'ky A., Byakova A., Podrezov Yu., Danylenko M. Nano crystallizations in Iron Alloys Induced by Friction Treatment and Nitrogen Diffusion. In Book «Metallic Materials with High Structural Efficiency».-Netherlands: Kluver Academic Publishers, 2004.-pp. 113-118.

[24] Yurkova A., Belots'ky A., Byakova A. Anomalous Nitrogen Solubility in Gradient Nanostructured Layer Formed in the Surface of Bulk Iron by Severe Plastic Deformation under the Friction. "Nanostructured Materials by High-Pressure Severe Plastic Deformation".-In Book NATO Science Series. II. Mathematics, Physics and Chemistry. Netherlands: Springer.2005.-Vol. 212. pp. 107-112.

[25] Ruban I. Automation of Laboratory and Bench Tests Processes in Tribotechnology/I. Ruban, O. Troshin, S. Smirnov et al.//Weapon Inventory and Military Hardware.Kharkiv: 2010.-No. 3 (23).-pp. 150-153.

[26] Tigetov D. G., Goritckiy U.A. Markov model of mechanical interaction of rough surfaces in the process of friction/D. G. Tigetov, U.A. Goritckiy//Friction and lubrication in machines and mechanisms.-2010.-№3.-P. 3-12.

[27] Volkov A., G. Physics course. Quantum physics: a textbook/A. G. Volkov, A. A. Povzner.-Ekateriburg: UrPhU, 2017.-155 p.

[28] Yezhov Yu. On Critical Loading Rate of Heterogeneous Materials at Erosion/Friction, Wear, Lubrication.//Yu. Yezhov, L. Pogodaev, Yu. Borschevskiy.-2013. Vol. 15 No. 57. [Electron. resource].-Access: http://www.tribo.ru.

[29] Goloskokov E. Elastic Acoustic Problems of Dynamics of Three-Layer Structures/E. Goloskokov, S. Beshenkov.Harkov: Vyshcha Shkola, 1980.-p. 120.

[30] Introduction to Superstring Theory, E. Kiritsis, Leuven Univ. Press, 1998 (Leuven Notes in Mathematical and Theoretical Physics, Vol. 9). 